Annals of Pure and Applied Mathematics

Vol. 19, No. 1, 2019, 37-51

ISSN: 2279-087X (P), 2279-0888(online)

Published on 12 February 2019

www.researchmathsci.org

DOI: http://dx.doi.org/10.22457/apam.597v19n1a5

Annals of

Pure and Applied

Mathematics

\title{
Pseuduo-Complementation on Almost Lattices
}

\author{
G.Nanaji Rao ${ }^{1}$ and R.Venkata Aravinda Raju ${ }^{2}$
}

${ }^{1}$ Department of Mathematics, Andhra University, Visakhapatnam-530003

Andhra Pradesh, India. Email: nani6us@yahoo.com

${ }^{2}$ Corresponding author. Email: aravindaraju.1@gmail.com

Received 2 January 2019; accepted 10 February 2019

\begin{abstract}
The concept of a pseudo-complementation $*$ on an almost lattice (AL) with 0 is introduced and proved some basic properties of pseudo-complementation *. Also, proved that pseudo-complementation $*$ on an $\mathrm{AL} L$ is equationally definable. A one-to-one correspondence between the set of pseudo-complementations on an AL $L$ with 0 and the set of all maximal elements of $L$ is obtained. It is also proved that $L^{*}=\left\{a^{*}: a \in L\right\}$ is a Boolean algebra which is independent (upto isomorphism) of the pseudocomplementation $*$ on $L$.
\end{abstract}

Keywords: almost lattice, pseudo-complementation, equationally definable class, Boolean algebra, maximal element.

AMS Mathematics Subject classification (2000): 06D99, 06D15

\section{Introduction}

It was Garett Birkhoff's (1911-1996) work in the mid thirties that started the general development of the lattice theory. In a brilliant series of papers, he demonstrated the importance of the lattice theory and showed that it provides a unified frame work for unrelated developments in many mathematical disciplines. Glivenko, Menger, Neumann, Ore, Gratzer, Halmos, Schmidt, Szasz, Stone, Dilworth and many others have developed enough of this field for making it attractive to the mathematicians and for its further progress. The traditional approach to lattice theory, which proceeds from partially ordered sets to general lattices, semimodular lattices, modular lattices and finally to distributive lattices. In [11], Rao and Sujatha introduced the concept of pseudocomplementation on Almost semilattice (ASL) and proved several properties of pseudocomplementation on ASL. In [10], Rao and Habtamu introduced the concept of an Almost Lattices (AL) as a common abstraction of most of the existing lattice theoretic generalizations of a Boolean algebra.

In this paper, we introduced the concept of pseudo-complementation $*$ on an $\mathrm{AL}$ with 0 and proved that it is equationally definable. It is observed that an AL with 0 can 


\section{G.Nanaji Rao and R.Venkata Aravinda Raju}

have more than one pseudo-complementation. In fact, if there is a pseudocomplementation on an AL $L$ with 0 , then we proved that each maximal element of $L$ gives rise to a pseudo-complementation and this correspondence is one-to-one. For any pseudo-complementation $*$ on an AL $L$ with 0 , we proved that the set $L^{*}=\left\{a^{*}: a \in L\right\}$ is a Boolean algebra, which is independent (upto isomorphism) of the pseudocomplementation $*$.

\section{Preliminaries}

In this section, we collect few important definitions and results which are already known and which will be used more frequently in the paper.

Definition 2.1. Let $(P, \leq)$ be a poset. Then $P$ is said to be a lattice ordered set if for every pair $x, y$ in $P$, l.u.b $\{x, y\}$ and g.l.b $\{x, y\}$ exist.

Definition 2.2. An algebra $(L, \vee, \wedge)$ of type $(2,2)$ is called a lattice if it satisfies the following axioms. For any $x, y, z \in L$,

(1) $x \vee y=y \vee x$ and $x \wedge y=y \wedge x$.

(Commutative Law)

(2) $(x \vee y) \vee z=x \vee(y \vee z)$ and $(x \wedge y) \wedge z=x \wedge(y \wedge z)$ (Associative Law)

(3) $x \vee(x \wedge y)=x$ and $x \wedge(x \vee y)=x$

(Absorption Law)

It can be easily seen that in any lattice $(L, \vee, \wedge), x \vee x=x$ and $x \wedge x=x$ (Idempotent Law).

Theorem 2.3. Let $(L, \leq)$ be a lattice ordered set. If we define $x \wedge y$ is the g.l.b of $\{x, y\}$ and $x \vee y$ is the l.u. $b$ of $\{x, y\}(x, y \in L)$, then $(L, \vee, \wedge)$ is a lattice.

Theorem 2.4. Let $(L, \vee, \wedge)$ be a lattice. If we define a relation $\leq$ on $L$, by $x \leq y$ if and only if $x=x \wedge y$, or equivalently $x \vee y=y$. Then $(L, \leq)$ is a lattice ordered set.

Note that theorems 2.3 and 2.4 together imply that the concepts of lattice and lattice ordered set are equivalent. We refer to it as a lattice in future.

Definition 2.5. Let $L$ be a lattice. Then $L$ is said to be bounded lattice if $L$ is bounded as a poset. That is, there exists $0,1 \in L$ such that $0 \wedge a=0$ and $1 \vee a=1$ for all $a \in L$.

Theorem 2.6. In any lattice $L$, the following are equivalent:

(1) $x \wedge(y \vee z)=(x \wedge y) \vee(x \wedge z)$

(2) $(x \vee y) \wedge z=(x \wedge z) \vee(y \wedge z)$

(3) $x \vee(y \wedge z)=(x \vee y) \wedge(x \vee z)$

(4) $(x \wedge y) \vee z=(x \vee z) \wedge(y \vee z)$. 
Pseuduo-Complementation on Almost Lattices

Definition 2.7. A lattice $L$ is called a distributive lattice if it satisfies any one of the above four conditions.

Theorem 2.8. Let $L$ be a lattice. Then for any $x, y, z \in L$, the following conditions are equivalent:

(1) $x \vee(y \wedge z)=(x \vee y) \wedge(x \vee z)$

(2) $x \wedge(y \vee z)=(x \wedge y) \vee(x \wedge z)$

(3) $(x \vee y) \wedge z \leq x \vee(y \wedge z)$

Definition 2.9. A bounded lattice $L$ with bounds 0 and 1 is said to be complemented if to each $x \in L$, there exists $y \in L$ such that $x \wedge y=0$ and $x \vee y=1$.

Definition 2.10. A complemented distributive lattice is called a Boolean algebra.

Definition 2.11. Let $B_{1}$ and $B_{2}$ be two Boolean algebras. A mapping $f: B_{1} \rightarrow B_{2}$ is called a Boolean homomorphism, if it is a lattice homomorphism and preserves complementations. That is, for any $a, b \in B_{1} ; f(a \vee b)=f(a) \vee f(b), f(a \wedge b)=$ $f(a) \wedge f(b)$ and $f\left(a^{\prime}\right)=(f(a))^{\prime}$.

It can be easily observed that, if $f$ is a lattice homomorphism from $B_{1}$ to $B_{2}$, then $f(0)$ $=0$ and $f(1)=1$ if and only if $f\left(a^{\prime}\right)=(f(a))^{\prime} \forall a \in B_{1}$.

Definition 2.12. A Boolean homomorphism $f: B_{1} \rightarrow B_{2}$ is said to be Boolean isomorphism if $f$ is a bijection.

Definition 2.13. An Almost semilattice is an algebra $(L, \circ)$, where $L$ is a non-empty set and $\circ$ is a binary operation on $L$ satisfies the following conditions:

(1) $x \circ(y \circ z)=(x \circ y) \circ z \quad$ (Associative Law)

(2) $(x \circ y) \circ z=(y \circ x) \circ z \quad$ (Almost Commutative Law)

(3) $x \circ x=x$ for all $x, y, z \in L$. (Idempotent Law)

Definition 2.14. Let $(L, \circ, 0)$ be an almost semilattice with 0 . Then a unary operation $x \mapsto x^{*}$ on $L$ is said to be pseudo-complementation on $L$ if, for any $a, b \in L$, it satisfies the following condition:

(1) $x \circ y=0 \Rightarrow x^{*} \circ y=y$

(2) $x \circ x^{*}=0$

Lemma 2.15. Let $L$ be a PCASL. Then for any $a, b \in L$, we have the following: 


\section{G.Nanaji Rao and R.Venkata Aravinda Raju}
(1) $0^{*} \circ a=a$
(2) $0^{*}$ is unimaximal
(3) $a^{* *} \circ a=a$
(4) $a \circ a^{* * *}=0$
(5) $a \leq b \Rightarrow a^{*} \circ b^{*}=b^{*}$
(6) $a$ is unimaximal $\Rightarrow a^{*}=0$
(7) $0^{* *}=0$
(8) $a^{* *}$ is unimaximal $\Leftrightarrow a^{*}=0$
(9) $a=0 \Leftrightarrow a^{* *}=0$

Theorem 2.16. Let $L$ be an $A S L$ with 0 . Then a unary operation $*: L \rightarrow L$ is a pseudocomplementation on $L$ if and only if it satisfies the following conditions:
(1) $a^{*} \circ b=(a \circ b)^{*} \circ b$
(2) $0^{*} \circ a=a$
(3) $0^{* *}=0$

Definition 2.17. An algebra $(L, \vee, \wedge, 0)$ of type $(2,2,0)$ is called an AL with 0 if, for any $a, b, c \in L$, it satisfies the following conditions:
(1) $(a \wedge b) \wedge c=(b \wedge a) \wedge c$
(2) $(a \vee b) \wedge c=(b \vee a) \wedge c$
(3) $(a \wedge b) \wedge c=a \wedge(b \wedge c)$
(4) $(a \vee b) \vee c=a \vee(b \vee c)$
(5) $a \wedge(a \vee b)=a$
(6) $a \vee(a \wedge b)=a$
(7) $(a \wedge b) \vee b=b$
(8) $0 \wedge a=a$

Definition 2.18. Let $L$ be an AL with 0 . For any $a, b \in L$, define $a \leq b$ if and only if $a=a \wedge b$ or equivalently $a \vee b=b$. Then $\leq$ is a partial ordering on $\mathrm{L}$.

Lemma 2.19. Let $L$ be an AL with 0 . Then for any $a, b \in L$, we have the following:
(1) $a \wedge 0=0$
(2) $a \vee 0=a$
(3) $0 \vee a=a$
(4) $a \wedge b=0 \Leftrightarrow b \wedge a=0$
(5) $a \wedge b=b \wedge a$ whenever $a \wedge b=0$

Lemma 2.20. Let $L$ be an $\mathrm{AL}$ with 0 . For any $a, b, c \in L$, we have the following:

(1) $a \wedge a=a$ and $a \vee a=a$

(2) $a \wedge b=a \Leftrightarrow a \vee b=b$

(3) $a \wedge b=b \wedge a$ whenever $a \leq b$ 
Pseuduo-Complementation on Almost Lattices

(4) $a \leq a \vee b$ and $a \wedge b \leq b$

(5) $(a \vee b) \wedge a=a$ and $(a \vee b) \wedge b=b$

(6) $a \wedge b=b \Leftrightarrow a \vee b=a$

(7) $a \vee b=b \vee a$ whenever $a \leq b$

(8) $b \vee(a \wedge b)=b$

(9) $a \vee b=b \vee a \Rightarrow a \wedge b=b \wedge a$

(10) If $a \leq b$ and $b \leq c$, then $a \wedge b \leq c$ and $a \vee b \leq c$

(11) $(a \vee b) \vee b=a \vee b$

(12) $(a \vee b) \vee a=a \vee b$

(13) $a \vee(a \vee b)=a \vee b$

(14) $a \wedge(a \wedge b)=a \wedge b$

(15) $(a \wedge b) \wedge b=a \wedge b$

(16) $b \wedge(a \wedge b)=a \wedge b$

(17) $a \vee b=a \vee(b \vee a)$

(18) $a \wedge b=0 \Leftrightarrow b \wedge a=0$

Definition 2.21. An AL $L$ is said to be directed above if for any $a, b \in L$ there exists $c \in L$ such that $a, b \leq c$.

Theorem 2.22. Let $L$ be an AL. Then the following are equivalent:

(1) $L$ is directed above

(2) $\wedge$ is commutative

(3) $\vee$ is commutative

(4) $L$ is a lattice.

Definition 2.23. Let $L$ be an AL with 0 . Then an element $a \in L$ is said to be maximal if for any $x \in L, a \leq x$ implies $a=x$.

Lemma 2.24. Let $L$ be an AL with $0, m \in L$. Then the following are equivalent:

(1) $m$ is maximal with respect to the partial ordering $\leq$

(2) $m \vee x=m$ for all $x \in L$

(3) $m \wedge x=x$ for all $x \in L$

Definition 2.25. Let $L$ be any nonempty set. Define for any $x, y \in L, x \vee y=x=y \wedge$ $x$. Then, clearly $L$ is an AL and is called discrete AL.

Definition 2.26. A ring $R$ is called a regular ring if to each $a \in L$, there exists $x \in R$ such that $a \times a=a$. 


\section{G.Nanaji Rao and R.Venkata Aravinda Raju}

\section{Pseudo-complementation}

In this section, we introduce the concept of pseudo-complementation on an almost lattice with 0 and prove some basic properties of an $\mathrm{AL}$ with pseudo-complementation and we prove that an $\mathrm{AL}$ with pseudo-complementation is equationally definable.

Definition 3.1. Let $L$ be an AL with 0 . Then a unary operation $a \mapsto a^{*}$ on $L$ is called a pseudo-complementation on $L$ if, for any $a, b \in L$, it satisfies the following conditions:

$\left(P_{1}\right) a \wedge b=0 \Rightarrow a^{*} \wedge b=b$

$\left(P_{2}\right) a \wedge a^{*}=0$

$\left(P_{3}\right)(a \vee b)^{*}=a^{*} \wedge b^{*}$

We first observe that $\left(P_{1}\right),\left(P_{2}\right)$ and $\left(P_{3}\right)$ are independent.

Example 3.2. Let $X$ be a discrete AL with 0 and with atleast two elements, say 1,2 other than 0 . Then $\left(X^{3}, \vee, \wedge, 0\right)$ is an $\mathrm{AL}$ with 0 , where $\vee, \wedge$ are defined coordinate wise. Now, for any $x \in X^{3}$, we write $|x|$ for the number of non-zero entries in $x$. Define * on $X^{3}$ as follows: For any $x \in X^{3}$ and $i=1,2,3$.

$$
x_{i}^{*}=\left\{\begin{array}{l}
0 \quad \text { if } x_{i} \neq 0 \\
1 \quad \text { if } x_{i}=0 \text { and }|x|=1 \text { and } 0^{*}=(2,2,2) \\
2 \quad \text { if } x_{i}=0 \text { and }|x|>1
\end{array}\right.
$$

then $\left(X^{3}, \vee, \wedge, 0\right)$ is an AL with $(0,0,0)$ as 0 which satisfies $\left(P_{1}\right)$ and $\left(P_{2}\right)$ but not $\left(P_{3}\right)$. (If $a=(1,0,0)$ and $b=(0,1,0)$, then $a^{*}=(0,1,1), b^{*}=(1,0,1)$ and $a \vee$ $b=(1,1,0)$, so $(a \vee b)^{*}=(0,0,2)$ and $\left.a^{*} \wedge b^{*}=(0,0,1)\right)$.

Example 3.3. Let $L$ be an AL with 0 with at least two elements. Define $a^{*}=0$ for all $a \in L$. Then $L$ satisfies $\left(P_{2}\right)$ and $\left(P_{3}\right)$, but not $\left(P_{1}\right)($ If $0 \neq b \in L$, then $0 \wedge b=$ 0 and $0^{*} \wedge b=0 \neq b$ )

Example 3.4. Let $L$ be a bounded distributive lattice with bounds $0 \neq 1$. Now, for any $a \in L$, define $a^{*}=1$ for all $a \in L$. Then $L$ satisfies $\left(P_{1}\right)$ and $\left(P_{3}\right)$ but not $\left(P_{2}\right)$.

In the following we give certain examples of pseudo-complemented ALs which are not pseudo-complemented lattices.

Example 3.5. Let $L=\{0, a, b, c\}$ and define $\wedge$ and $\vee$ on $L$ as follows:

\begin{tabular}{|c|c|c|c|c|}
\hline $\mathrm{V}$ & $\mathbf{0}$ & $\mathbf{a}$ & $\mathbf{b}$ & $\mathbf{c}$ \\
\hline $\mathbf{0}$ & $\mathbf{0}$ & $\mathbf{a}$ & $\mathbf{b}$ & $\mathbf{c}$ \\
\hline $\mathbf{a}$ & $\mathbf{a}$ & $\mathbf{a}$ & $\mathbf{a}$ & $\mathbf{a}$ \\
\hline $\mathbf{b}$ & $\mathbf{b}$ & $\mathbf{b}$ & $\mathbf{b}$ & $\mathbf{b}$ \\
\hline $\mathbf{c}$ & $\mathbf{c}$ & $\mathbf{a}$ & $\mathbf{b}$ & $\mathbf{c}$ \\
\hline
\end{tabular}

\begin{tabular}{|l|l|l|l|l|}
\hline$\wedge$ & $\mathbf{0}$ & $\mathbf{a}$ & $\mathbf{b}$ & $\mathbf{c}$ \\
\hline $\mathbf{0}$ & $\mathbf{0}$ & $\mathbf{0}$ & $\mathbf{0}$ & $\mathbf{0}$ \\
\hline $\mathbf{a}$ & $\mathbf{0}$ & $\mathbf{a}$ & $\mathbf{b}$ & $\mathbf{b}$ \\
\hline $\mathbf{b}$ & $\mathbf{0}$ & $\mathbf{a}$ & $\mathbf{b}$ & $\mathbf{c}$ \\
\hline $\mathbf{c}$ & $\mathbf{0}$ & $\mathbf{c}$ & $\mathbf{c}$ & $\mathbf{c}$ \\
\hline
\end{tabular}


Pseuduo-Complementation on Almost Lattices

and define $x^{*}=0$ if $x \neq 0$ and $0^{*}=b$. Then $(L, \vee, \wedge, 0)$ is an AL with 0 and $x \mapsto x^{*}$ is a pseudo-complementation on $L$. Note that $(L, \vee, \wedge)$ is not a lattice.

Example 3.6. Let $(R,+, ., 0)$ be a commutative regular ring. To each $a \in R$, let $a^{0}$ be the unique idempotent element in $R$ such that $a R=a^{0} R$. Define, for any $a, b \in R$,

(1) $a \wedge b=a^{0} b$

(2) $a \vee b=a+\left(1-a^{0}\right) b$

(3) $a^{*}=1-a^{0}$

then $(R, \vee, \wedge, 0)$ is an AL with 0 and $*$ is a pseudo-complementation on $R$.

In the case of distributive lattice with 0 , it is well known that the element $a^{*}$ satisfying the properties $\left(P_{1}\right)$ and $\left(P_{2}\right)$ is unique (if exists) and that $\left(P_{3}\right)$ is a consequence of $\left(P_{1}\right)$ and $\left(P_{2}\right)$ and hence, there can be atmost one pseudocomplementation. However, in an $\mathrm{AL}$ with 0 , there can be several pseudocomplementations; for, consider the following examples.

Example 3.7. Let $X$ be a discrete AL with 0 . For any $x \neq 0$ in $X$, define for any $a \in X$,

$$
a^{*}= \begin{cases}0 & \text { if } a \neq 0 \\ x & \text { if } a=0\end{cases}
$$

then $*$ is a pseudo-complementation on $X$. Here, with each $x \neq 0$ in $X$, we obtain a pseudo-complementation on $(X, \vee, \wedge, 0)$. More generally, we have the following:

Example 3.8. Let $X$ be a non-empty set with at least two elements and let $Y$ be any set and $f_{0} \in X^{Y}$. Now, for any $a, b \in X^{Y}$, define

$$
\begin{aligned}
& (a \vee b)(y) \\
& = \begin{cases}a(y) \text { if } a(y) \neq f_{0}(y) \\
b(y) \text { if } a(y)=f_{0}(y) ;\end{cases} \\
& (a \wedge b)(y)= \begin{cases}b(y) \text { if } a(y) \neq f_{0}(y) \\
f_{0}(y) \text { if } a(y)=f_{0}(y) .\end{cases}
\end{aligned}
$$

Then $\left(X^{Y}, \vee, \wedge, f_{0}\right)$ is an $\mathrm{AL}$ with $f_{0}$ as zero element. Now, let $f \in X^{Y}$ such that $f(y) \neq f_{0}(y)$ for all $y \in Y$. For any $a \in X^{Y}$, define

$$
a^{f}(y)=\left\{\begin{array}{l}
f_{0}(y) \text { if } a(y) \neq f_{0}(y) \\
f(y) \text { if } a(y)=f_{0}(y)
\end{array}\right.
$$

Then $a \mapsto a^{f}$ is a pseudo-complementation on $X^{Y}$ and, conversely, if $a \mapsto a^{*}$ is pseudo-complementation on $X^{Y}$, then there exists $f \in X^{Y}$ such that $f(y) \neq f_{0}(y)$ for all $y \in Y$ and $a^{*}=a^{f}$ for all $a \in X^{Y}$ (take $f=f_{0}^{*}$ )

In the following, we prove some basic properties of pseudo-complementation $*$ on an AL $L$. 


\section{G.Nanaji Rao and R.Venkata Aravinda Raju}

Lemma 3.9. Let $L$ be an $\mathrm{AL}$ with 0 and $*$ a pseudo-complementation on $L$. Then, for any $a, b \in L$, we have the following:

(1) $0^{*}$ is maximal

(2) If a is maximal, then $a^{*}=0$

(3) $0^{* *}=0$

(4) $a^{*} \wedge a=0$

(5) $a^{* *} \wedge a=a$

(6) $a^{*}=a^{* * *}$

(7) $a^{*}=0 \Leftrightarrow a^{* *}$ is maximal

(8) $a^{*} \leq 0^{*}$

(9) $a^{*} \wedge b^{*}=b^{*} \wedge a^{*}$

(10) $(a \vee b)^{*}=(b \vee a)^{*}$

(11) $a \leq b \Rightarrow b^{*} \leq a^{*}$

(12) $a^{*} \leq(a \wedge b)^{*}$ and $b^{*} \leq(a \wedge b)^{*}$

(13) $(a \vee b)^{*} \leq a^{*}$ and $(a \vee b)^{*} \leq b^{*}$

(14) $a^{*} \leq b^{*} \Leftrightarrow b^{* *} \leq a^{* *}$

(15) $a=0 \Leftrightarrow a^{* *}=0$

Proof: (1) For any $a \in L$, we have $0 \wedge a=0$, and hence $0^{*} \wedge a=a$. It follows that $0^{*}$ is maximal.

(2) Suppose $a$ is maximal. Since, $a \leq a \vee a^{*}, a=a \vee a^{*}$. Hence $a^{*}=$ $\left(a \vee a^{*}\right)^{*}=a^{*} \wedge a^{* *}=0$

(3) Proof follows from (1) and (2).

(4) Proof follows from lemma 2.20(18).

(5) By (4), we have $a^{*} \wedge a=0$. This implies $a^{* *} \wedge a=a$.

(6) From (5) we have $a^{* *} \wedge a=a$. This implies $a^{* *}=a^{* *} \vee a$. Hence $a^{* * *}=\left(a^{* *} \vee a\right)^{*}=a^{* * *} \wedge a^{*}=a^{*}$. Thus $a^{*}=a^{* * *}$.

(7) Proof follows from (1), (2) and (6).

(8) $a^{*}=(a \vee 0)^{*}=a^{*} \wedge 0^{*}$, and hence, $a^{*} \leq 0^{*}$.

(9) From (8), $a^{*}, b^{*} \leq 0^{*}$. Therefore, $a^{*} \wedge b^{*}=b^{*} \wedge a^{*}$.

(10) Proof follows by (9).

(11) Suppose $a \leq b$. Then $b=a \vee b$.

This implies $b^{*}=(a \vee b)^{*}=a^{*} \wedge b^{*}=b^{*} \wedge a^{*}$. Therefore $b^{*} \leq a^{*}$.

(12) We have $a=a \vee(a \wedge b)$. Therefore, $a^{*}=a^{*} \wedge(a \wedge b)^{*}$, and hence, $a^{*} \leq(a \wedge b)^{*}$. Also, we have $a \wedge b \leq b$. This implies $b^{*} \leq(a \wedge b)^{*}$.

(13) We have $a \leq a \vee b$ and $b \leq b \vee a$.

This implies $(a \vee b)^{*} \leq a^{*}$ and $(a \vee b)^{*}=(b \vee a)^{*} \leq b^{*}$.

(14) Proof follows from (6) and (11).

(15) Suppose $a^{* *}=0$. Now, $0=0 \wedge a=a^{* *} \wedge a=a$. Thus $a=0$. Converse follows from condition (3). 


\section{Pseuduo-Complementation on Almost Lattices}

Lemma 3.10. Let $L$ be an $\mathrm{AL}$ with 0 and $*$ a pseudo-complementation on $L$. Then, for any $a, b \in L$, the following are equivalent:

(1) $a \wedge b=0$

(2) $a^{* *} \wedge b=0$

(3) $a^{* *} \wedge b^{* *}=0$

(4) $a \wedge b^{* *}=0$

Proof: (1) $\Rightarrow$ (2): Suppose $a \wedge b=0$. Then $a^{*} \wedge b=b$. Now, consider $a^{* *} \wedge$ $b=a^{* *} \wedge\left(a^{*} \wedge b\right)=\left(a^{* *} \wedge a^{*}\right) \wedge b=0 \wedge b=0$. Thus $a^{* *} \wedge b=0$.

(2) $\Rightarrow$ (3): Suppose $a^{* *} \wedge b=0$. Then $b \wedge a^{* *}=0$. This implies $b^{*} \wedge a^{* *}=$ $a^{* *}$. Now, consider $a^{* *} \wedge b^{* *}=\left(b^{*} \wedge a^{* *}\right) \wedge b^{* *}=\left(a^{* *} \wedge b^{*}\right) \wedge b^{* *}=a^{* *} \wedge$ $\left(b^{*} \wedge b^{* *}\right)=a^{* *} \wedge 0=0$. Thus $a^{* *} \wedge b^{* *}=0$.

(3) $\Rightarrow$ (4): Suppose $a^{* *} \wedge b^{* *}=0$. Now, consider $a \wedge b^{* *}=\left(a^{* *} \wedge a\right) \wedge b^{* *}=$ $\left(a \wedge a^{* *}\right) \wedge b^{* *}=a \wedge\left(a^{* *} \wedge b^{* *}\right)=a \wedge 0=0$. Thus $a \wedge b^{* *}=0$.

(4) $\Rightarrow$ (1): Suppose $a \wedge b^{* *}=0$. Now, consider, $a \wedge b=a \wedge\left(b^{* *} \wedge b\right)=$ $\left(a \wedge b^{* *}\right) \wedge b=0 \wedge b=0$. Therefore $a \wedge b=0$.

Lemma 3.11. Let $L$ be an AL with 0 and * a pseudo-complementation on $L$. Then, for any $a, b \in L$, the following hold:

(1) $(a \wedge b)^{* *}=a^{* *} \wedge b^{* *}$

(2) $(a \wedge b)^{*}=(b \wedge a)^{*}$

Proof: Let $a, b \in L$.

(1) Consider, $\left(a^{* *} \wedge b^{* *}\right) \wedge(a \wedge b)^{* *}=a^{* *} \wedge\left(b^{* *} \wedge(a \wedge b)^{* *}\right)$

$$
\begin{aligned}
& =a^{* *} \wedge\left(b^{*} \vee(a \wedge b)^{*}\right)^{*}\left(b y\left(P_{3}\right)\right) \\
& =a^{* *} \wedge(a \wedge b)^{* *} \\
& =\left(a^{*} \vee(a \wedge b)^{*}\right)^{*}\left(b y\left(P_{3}\right)\right) \\
& =(a \wedge b)^{* *}
\end{aligned}
$$

Again, we have $(a \wedge b) \wedge(a \wedge b)^{*}=0 \Rightarrow a \wedge\left(b \wedge(a \wedge b)^{*}\right)=0$

$$
\begin{aligned}
& \Rightarrow a^{* *} \wedge\left(b \wedge(a \wedge b)^{*}\right)=0 \\
& \Rightarrow\left(a^{* *} \wedge b\right) \wedge(a \wedge b)^{*}=0 \\
& \Rightarrow\left(b \wedge a^{* *}\right) \wedge(a \wedge b)^{*}=0 \\
& \Rightarrow b \wedge\left(a^{* *} \wedge(a \wedge b)^{*}\right)=0 \\
& \Rightarrow b^{* *} \wedge\left(a^{* *} \wedge(a \wedge b)^{*}\right)=0 \\
& \Rightarrow\left(b^{* *} \wedge a^{* *}\right) \wedge(a \wedge b)^{*}=0 \\
& \Rightarrow\left(a^{* *} \wedge b^{* *}\right) \wedge(a \wedge b)^{*}=0 \\
& \Rightarrow(a \wedge b)^{*} \wedge\left(a^{* *} \wedge b^{* *}\right)=0
\end{aligned}
$$

$\Rightarrow(a \wedge b)^{* *} \wedge\left(a^{* *} \wedge b^{* *}\right)=a^{* *} \wedge b^{* *}\left(\right.$ by $\left.\left(P_{1}\right)\right)$

It follows that, $(a \wedge b)^{* *}=a^{* *} \wedge b^{* *}$.

(2) $(a \wedge b)^{*}=(a \wedge b)^{* * *}$ 
G.Nanaji Rao and R.Venkata Aravinda Raju

$$
\begin{aligned}
& =\left(a^{* *} \wedge b^{* *}\right)^{*} \\
& =\left(b^{* *} \wedge a^{* *}\right)^{*} \\
& =(b \wedge a)^{* * *} \\
& =(b \wedge a)^{*}
\end{aligned}
$$

First, observe that if $L$ is an AL with 0 , does not satisfies the distributive law ( $x \vee$ y) $\wedge z=(x \wedge z) \vee(y \wedge z) \forall x, y, z$ in $L$ and $*$ is a unary operation on $L$ which satisfies the following conditions:

(1) $a \wedge a^{*}=0$

(2) $a^{* *} \vee a=a^{* *}$

(3) $(a \vee b)^{*}=a^{*} \wedge b^{*}$

(4) $(a \wedge b)^{* *}=a^{* *} \wedge b^{* *}$

(5) $0^{*} \wedge a=a$

does not imply $*$ is a pseudo-complementation on $L$. For, consider the following example.

Example 3.12. Let $L=\{0, a, b, c, d\}$ and define binary operations $\vee$ and $\wedge$ on $L$ as follows:

\begin{tabular}{|c|c|c|c|c|c|}
\hline $\mathrm{V}$ & 0 & $\mathrm{a}$ & $\mathrm{b}$ & $\mathrm{c}$ & $\mathrm{d}$ \\
\hline 0 & 0 & $\mathrm{a}$ & $\mathrm{b}$ & $\mathrm{c}$ & $\mathrm{d}$ \\
\hline $\mathrm{a}$ & $\mathrm{a}$ & $\mathrm{a}$ & $\mathrm{d}$ & $\mathrm{d}$ & $\mathrm{d}$ \\
\hline $\mathrm{b}$ & $\mathrm{b}$ & $\mathrm{d}$ & $\mathrm{b}$ & $\mathrm{d}$ & $\mathrm{d}$ \\
\hline $\mathrm{c}$ & $\mathrm{c}$ & $\mathrm{d}$ & $\mathrm{d}$ & $\mathrm{c}$ & $\mathrm{d}$ \\
\hline $\mathrm{d}$ & $\mathrm{d}$ & $\mathrm{d}$ & $\mathrm{d}$ & $\mathrm{d}$ & $\mathrm{d}$ \\
\hline
\end{tabular}

\begin{tabular}{|c|c|c|c|c|c|}
\hline$\wedge$ & 0 & $\mathrm{a}$ & $\mathrm{b}$ & $\mathrm{c}$ & $\mathrm{d}$ \\
\hline 0 & 0 & 0 & 0 & 0 & 0 \\
\hline $\mathrm{a}$ & 0 & $\mathrm{a}$ & 0 & 0 & $\mathrm{a}$ \\
\hline $\mathrm{b}$ & 0 & 0 & $\mathrm{~b}$ & 0 & $\mathrm{~b}$ \\
\hline $\mathrm{c}$ & 0 & 0 & 0 & $\mathrm{c}$ & $\mathrm{c}$ \\
\hline $\mathrm{d}$ & 0 & $\mathrm{a}$ & $\mathrm{b}$ & $\mathrm{c}$ & $\mathrm{d}$ \\
\hline
\end{tabular}

Then clearly $L$ is an AL with 0 . Now, define a unary operation $*$ on $L$ by $0^{*}=d, d^{*}=0$ and $a^{*}=b^{*}=c^{*}=0$. Then $*$ satisfies the conditions (1) to (5) above but, $*$ is not pseudo-complementation on $L$. Since, $a \wedge b=0$. But, $a^{*} \wedge b=0 \wedge b=0 \neq b$.

However, if an AL $L$ satisfies the distributive law $(x \vee y) \wedge z=(x \wedge z) \vee(y \wedge z)$ for all $x, y, z \in L$, then we prove that pseudo-complementation $*$ on an $\operatorname{AL} L$ is equationally definable.

Theorem 3.13. Let $L$ be an AL with 0 which satisfies $(x \vee y) \wedge z=(x \wedge z) \vee$ $(y \wedge z)$ for all $x, y, z \in L$. Then a unary operation $a \mapsto a^{*}$ on $L$ is a pseudocomplementation on $L$ if and only if, it satisfies the following conditions:

(1) $a \wedge a^{*}=0$

(2) $a^{* *} \vee a=a^{* *}$

(3) $(a \vee b)^{*}=a^{*} \wedge b^{*}$

(4) $(a \wedge b)^{* *}=a^{* *} \wedge b^{* *}$ 
Pseuduo-Complementation on Almost Lattices

(5) $0^{*} \wedge a=a$

Proof: Assume that $*$ satisfies conditions (1) to (5). Then we have $a \wedge a^{*}=0$ and $(a \vee b)^{*}=a^{*} \wedge b^{*}$. Let $a, b \in L$ such that $a \wedge b=0$. Then

$$
\begin{aligned}
b & =b^{* *} \wedge b \\
& =0^{*} \wedge\left(b^{* *} \wedge b\right) \\
& =\left(a^{*} \wedge a^{* *}\right)^{*} \wedge\left(b^{* *} \wedge b\right) \\
& =\left(a \vee a^{*}\right)^{* *} \wedge\left(b^{* *} \wedge b\right) \\
& =\left(\left(a \vee a^{*}\right)^{* *} \wedge b^{* *}\right) \wedge b \\
& =\left(\left(a \vee a^{*}\right) \wedge b\right)^{* *} \wedge b \\
& =\left((a \wedge b) \vee\left(a^{*} \wedge b\right)\right)^{* *} \wedge b \\
& =\left(a^{*} \wedge b\right)^{* *} \wedge b \\
& =\left(a^{* * *} \wedge b^{* *}\right) \wedge b \\
& =a^{* * *} \wedge\left(b^{* *} \wedge b\right) \\
& =a^{* * *} \wedge b \\
& =\left(a^{* * *} \wedge a^{*}\right) \wedge b(b y(2)) \\
& =a^{*} \wedge b
\end{aligned}
$$

Thus $*$ is a pseudo-complementation on $L$. Converse follows from the definition of pseudo-complementation and by Lemmas 3.9 and 3.11.

In the following we obtain a set of identities which characterise a pseudocomplementation on an AL with 0 .

Theorem 3.14. Let $L$ be an AL with 0 . Then a unary operation $a \mapsto a^{*}$ on $L$ is a pseudo-complementation on $L$ if and only if, it satisfies the following conditions:

(1) $a^{*} \wedge b=(a \wedge b)^{*} \wedge b$

(2) $0^{*} \wedge a=a$

(3) $0^{* *}=0$

(4) $(a \vee b)^{*}=a^{*} \wedge b^{*}$

Proof: Assume that $*$ satisfies the given condition. Let $a, b \in L$ such that $a \wedge b=0$. Then

$$
\begin{aligned}
a^{*} \wedge b & =(a \wedge b)^{*} \wedge b \\
& =0^{*} \wedge b=b \quad(b y(2))
\end{aligned}
$$

Again by (2), $a^{*} \wedge a=\left(0^{*} \wedge a\right)^{*} \wedge a=0^{* *} \wedge a=0 \wedge a=0$. It follows that, $a \wedge a^{*}=0$. Therefore $*$ is a pseudo-complementation on $L$.

Conversely, suppose $*$ is a pseudo-complementation on $L$. Then we have $0^{*} \wedge a=$ $a, 0^{* *}=0$ and $(a \vee b)^{*}=a^{*} \wedge b^{*}$. Now, we shall prove that $a^{*} \wedge b=(a \wedge b)^{*} \wedge b$. Now, we have

$$
\begin{aligned}
(a \wedge b) \wedge(a \wedge b)^{*}=0 & \Rightarrow a \wedge\left(b \wedge(a \wedge b)^{*}\right)=0 \\
& \Rightarrow a^{*} \wedge\left(b \wedge(a \wedge b)^{*}\right)=\left(b \wedge(a \wedge b)^{*}\right) \\
& \Rightarrow\left(a^{*} \wedge\left(b \wedge(a \wedge b)^{*}\right)\right) \wedge b=\left(b \wedge(a \wedge b)^{*}\right) \wedge b
\end{aligned}
$$


G.Nanaji Rao and R.Venkata Aravinda Raju

$$
\begin{aligned}
& \Rightarrow a^{*} \wedge\left(\left(b \wedge(a \wedge b)^{*}\right) \wedge b\right)=\left((a \wedge b)^{*} \wedge b\right) \wedge b \\
& \Rightarrow a^{*} \wedge\left((a \wedge b)^{*} \wedge(b \wedge b)\right)=(a \wedge b)^{*} \wedge(b \wedge b) \\
& \Rightarrow a^{*} \wedge\left((a \wedge b)^{*} \wedge b\right)=(a \wedge b)^{*} \wedge b \\
& \Rightarrow\left(a^{*} \wedge(a \wedge b)^{*}\right) \wedge b=(a \wedge b)^{*} \wedge b \\
& \Rightarrow\left((a \wedge b)^{*} \wedge a^{*}\right) \wedge b=(a \wedge b)^{*} \wedge b \\
& \Rightarrow(a \wedge b)^{*} \wedge\left(a^{*} \wedge b\right)=(a \wedge b)^{*} \wedge b \\
& \Rightarrow a^{*} \wedge b=(a \wedge b)^{*} \wedge b\left(\text { since }(a \wedge b) \wedge\left(a^{*} \wedge b\right)=0\right)
\end{aligned}
$$

\section{One-to-one correspondence}

In this section, we prove that, if $L$ is an $\mathrm{AL}$ with 0 and with pseudo-complementation *, the set $L^{*}=\left\{a^{*}: a \in L\right\}$ is a Boolean algebra. In section 3, it is remarked that an $\mathrm{AL}$ with 0 can have more than one pseudo-complementation and examples were given to this effect. We prove that if $L$ is an AL with a pseudo-complementation *, then to each maximal element $m$ in $L$, we obtain a pseudo-complementation $*_{m}$. In fact, we prove that this correspondence between the set of all maximal elements in $\mathrm{L}$ and set of all pseudocomplementation $*$ on $L$ is one-to-one and that the Boolean algebra $L^{*}$ is independent (upto isomorphism) of the pseudo-complementation *.

Theorem 4.1. Let $L$ be an AL with 0 and $*$ be a pseudo-Complementation on $L$. For any $a^{*}, b^{*} \in L^{*}$, define $a^{*} \leq b^{*}$ if and only if $a^{*} \wedge b^{*}=a^{*}$. Then, $\left(L^{*}, \leq\right)$ is a Boolean algebra, in which $\left(a^{* *} \wedge b^{* *}\right)^{*}$ is the least upper bound (l.u.b) and $a^{*} \wedge b^{*}$ is the greatest lower bound (g.l.b) of $a^{*}$ and $b^{*}$ in $L^{*}$.

Proof: Clearly, $\leq$ is a partial ordering on $L^{*}$. Let $a^{*}, b^{*} \in L^{*}$. Then $a^{*} \wedge b^{*}=$ $(a \vee b)^{*} \in L^{*}$ and clearly $a^{*} \wedge b^{*}$ is the g.l.b of $\left\{a^{*}, b^{*}\right\}$ in $L^{*}$. Now, we have $a^{* *} \wedge b^{* *} \leq b^{* *}$. This implies that $b^{*}=b^{* * *} \leq\left(a^{* *} \wedge b^{* *}\right)^{*}$. Similarly, we get $a^{*} \leq\left(a^{* *} \wedge b^{* *}\right)^{*}$. Therefore $\left(a^{* *} \wedge b^{* *}\right)^{*}$ is an upper bound of $\left\{a^{*}, b^{*}\right\}$ in $L^{*}$. Suppose $c^{*} \in L^{*}$ such that $c^{*}$ is an upper bound of $\left\{a^{*}, b^{*}\right\}$. Then $a^{*} \leq c^{*}$ and $b^{*} \leq$ $c^{*}$. Hence $c^{* *} \leq a^{* *}$ and $c^{* *} \leq b^{* *}$. This implies $c^{* *} \leq a^{* *} \wedge b^{* *}$. Therefore $\left(a^{* *} \wedge\right.$ $\left.b^{* *}\right)^{*} \leq c^{* * *}=c^{*}$ and hence, $\left(a^{* *} \wedge b^{* *}\right)^{*}$ is the l.u. $b$ of $\left\{a^{*}, b^{*}\right\}$ in $L^{*}$. We denote this l.u. $b$ of $\left\{a^{*}, b^{*}\right\}$ by $a^{*} \underline{\vee} b^{*}$. Therefore $\left(L^{*}, \underline{\vee}, \wedge\right)$ is a lattice.

Clearly, 0 and $0^{*}$ are the least and greatest elements in $L^{*}$, respectively. Hence $L^{*}$ is a bounded lattice. Let $a^{*} \in L^{*}$. Then, we have $a^{* *} \in L^{*}$ and $a^{*} \wedge a^{* *}=0$. Now, consider $a^{*} \underline{\vee} a^{* *}=\left(a^{* *} \wedge a^{* * *}\right)^{*}=0^{*}$. Thus every element in $L^{*}$ has a complement in $L^{*}$. Therefore $L^{*}$ is a complemented lattice. Now, we shall prove $L^{*}$ is distributive lattice. For, this it is enough to prove that for any $a^{*}, b^{*}, c^{*} \in L^{*},\left(a^{*} \underline{\vee} b^{*}\right) \wedge c^{*} \leq$ $a^{*} \underline{\vee}\left(b^{*} \wedge c^{*}\right)$ (see theorem 2.8.). Let $a^{*}, b^{*}, c^{*} \in L^{*}$. Then we have $\left(b^{*} \wedge c^{*}\right) \wedge$ $\left(a^{* *} \wedge\left(b^{*} \wedge c^{*}\right)^{*}\right)=0$. This implies $b^{*} \wedge\left(c^{*} \wedge\left(a^{* *} \wedge\left(b^{*} \wedge c^{*}\right)^{*}\right)\right)=0$. It follows that $b^{* *} \wedge\left(c^{*} \wedge\left(a^{* *} \wedge\left(b^{*} \wedge c^{*}\right)^{*}\right)\right)=\left(c^{*} \wedge\left(a^{* *} \wedge\left(b^{*} \wedge c^{*}\right)^{*}\right)\right)$. Hence we get $\left(c^{*} \wedge\left(a^{* *} \wedge\left(b^{*} \wedge c^{*}\right)^{*}\right)\right) \wedge b^{* *}=\left(c^{*} \wedge\left(a^{* *} \wedge\left(b^{*} \wedge c^{*}\right)^{*}\right)\right)$. Therefore $\left(c^{*} \wedge\right.$ 
Pseuduo-Complementation on Almost Lattices

$\left.\left(a^{* *} \wedge\left(b^{*} \wedge c^{*}\right)^{*}\right)\right) \leq b^{* *}$. Again, we have $a^{*} \wedge\left(c^{*} \wedge\left(a^{* *} \wedge\left(b^{*} \wedge c^{*}\right)^{*}\right)\right)=0$. This implies $a^{* *} \wedge\left(c^{*} \wedge\left(a^{* *} \wedge\left(b^{*} \wedge c^{*}\right)^{*}\right)\right)=\left(c^{*} \wedge\left(a^{* *} \wedge\left(b^{*} \wedge c^{*}\right)^{*}\right)\right)$. Hence we get $\left(c^{*} \wedge\left(a^{* *} \wedge\left(b^{*} \wedge c^{*}\right)^{*}\right)\right) \wedge a^{* *}=\left(c^{*} \wedge\left(a^{* *} \wedge\left(b^{*} \wedge c^{*}\right)^{*}\right)\right)$. Therefore $\left(c^{*} \wedge\left(a^{* *} \wedge\left(b^{*} \wedge c^{*}\right)^{*}\right)\right) \leq a^{* *}$. It follows that, $\left(c^{*} \wedge\left(a^{* *} \wedge\left(b^{*} \wedge c^{*}\right)^{*}\right)\right) \leq$ $a^{* *} \wedge b^{* *}$. This implies $\left(c^{*} \wedge\left(a^{* *} \wedge\left(b^{*} \wedge c^{*}\right)^{*}\right)\right) \wedge\left(a^{* *} \wedge b^{* *}\right)^{*} \leq\left(a^{* *} \wedge b^{* *}\right) \wedge$ $\left(a^{* *} \wedge b^{* *}\right)^{*}$. Hence we get $\left(c^{*} \wedge\left(a^{* *} \wedge\left(b^{*} \wedge c^{*}\right)^{*}\right)\right) \wedge\left(a^{* *} \wedge b^{* *}\right)^{*}=0$. Therefore $\left(\left(a^{* *} \wedge\left(b^{*} \wedge c^{*}\right)^{*}\right) \wedge c^{*}\right) \wedge\left(a^{* *} \wedge b^{* *}\right)^{*}=0$ and hence $\left(\left(a^{* *} \wedge\right.\right.$ $\left.\left.\left(b^{*} \wedge c^{*}\right)^{*}\right)\right) \wedge\left(c^{*} \wedge\left(a^{* *} \wedge b^{* *}\right)^{*}=0\right.$. It follows that $\left(\left(a^{* *} \wedge\left(b^{*} \wedge c^{*}\right)^{*}\right)\right)^{*} \wedge$ $\left(c^{*} \wedge\left(a^{* *} \wedge b^{* *}\right)^{*}\right)=\left(c^{*} \wedge\left(a^{* *} \wedge b^{* *}\right)^{*}\right) \quad$ Hence $\quad\left(c^{*} \wedge\left(a^{* *} \wedge b^{* *}\right)^{*}\right) \wedge\left(\left(a^{* *} \wedge\right.\right.$ $\left.\left.\left(b^{*} \wedge c^{*}\right)^{*}\right)\right)^{*}=\left(c^{*} \wedge\left(a^{* *} \wedge b^{* *}\right)^{*} \quad\right.$.Therefore $\quad\left(c^{*} \wedge\left(a^{* *} \wedge b^{* *}\right)^{*}\right) \leq$ $\left(\left(a^{* *} \wedge\left(b^{*} \wedge c^{*}\right)^{*}\right)\right)^{*}$. This implies $\left(\left(a^{* *} \wedge b^{* *}\right)^{*} \wedge c^{*}\right) \leq\left(\left(a^{* *} \wedge\left(b^{*} \wedge\right.\right.\right.$ $\left.\left.\left.c^{*}\right)^{*}\right)\right)^{*}$. Thus $\left(a^{*} \underline{\vee} b^{*}\right) \wedge c^{*} \leq a^{*} \underline{\vee}\left(b^{*} \wedge c^{*}\right)$. Therefore by theorem 2.8, $\left(L^{*}, \underline{\mathrm{v}}\right.$, $\wedge)$ is a distributive lattice. Thus $\left(L^{*}, \underline{\vee}, \wedge, 0,0^{*}\right)$ is a Boolean algebra.

Corollary 4.2. Let $L$ be pseudo-complemented AL with pseudo-complementation *. Then the map $f: L \rightarrow L^{*}$ defined by $f(a)=a^{* *}$ is an epimorphism.

Proof: Clearly, $f$ is well-defined. Let $a, b \in L$. Then, Let $f(a \vee b)=(a \vee b)^{* *}=$ $\left(a^{*} \wedge b^{*}\right)^{*}=\left(a^{* * *} \wedge b^{* * *}\right)^{*}=a^{* *} \underline{\vee} b^{* *}=f(a) \underline{\vee} f(b)$ and $f(a \wedge b)=(a \wedge$ $b)^{* *}=a^{* *} \wedge b^{* *}=f(a) \wedge f(b)$. Let $a^{*} \in L^{*}$. Then we have $a^{*} \in L$ and $f\left(a^{*}\right)=$ $a^{* * *}=a^{*}$. Therefore $f$ is an epimorphism.

Next, we shall prove that if $L$ is an AL with pseudo-complementation *, then a oneto-one correspondence between set of all maximal elements in $L$ and set of all pseudocomplementations on an AL $L$. For, this first we need following lemma.

Lemma 4.3. Let $L$ be an AL with 0 and let $*$ and $\perp$ be two pseudo-complementations on $L$. Then, for any $a, b \in L$ we have the following:

$$
\begin{aligned}
& \text { 1) } a^{*} \wedge a^{\perp}=a^{\perp} \text { and } a^{*} \vee a^{\perp}=a^{*} \\
& \text { 2) } a^{* \perp}=a^{\perp \perp} \\
& \text { 3) } a^{*}=b^{*} \Leftrightarrow a^{\perp}=b^{\perp} \\
& \text { 4) } a^{*}=0 \Leftrightarrow a^{\perp}=0 \Leftrightarrow(a \wedge b=0 \Rightarrow b=0) \\
& \text { 5) } a^{\perp}=a^{*} \wedge 0^{\perp}
\end{aligned}
$$

Proof: 1) Since $a \wedge a^{\perp}=0, a^{*} \wedge a^{\perp}=a^{\perp}$. It follows that $a^{*} \vee a^{\perp}=a^{*}$.

2) $a^{* \perp}=\left(a^{*} \vee a^{\perp}\right)^{\perp}=\left(a^{\perp} \vee a^{*}\right)^{\perp}=a^{\perp \perp}$.

3) Suppose $a^{*}=b^{*}$. Then $a^{\perp}=a^{\perp \perp \perp}=a^{* \perp \perp}=b^{* \perp \perp}=b^{\perp \perp \perp}=b^{\perp}$. Thus $a^{\perp}=b^{\perp}$.

Similarly we can prove the converse. 


\section{G.Nanaji Rao and R.Venkata Aravinda Raju}

4) Suppose $a^{*}=0$. Now, we consider $a^{\perp}=a^{*} \wedge a^{\perp}=0 \wedge a^{\perp}=0$. Therefore $a^{\perp}=0$.

Suppose $a^{\perp}=0$ and suppose $a \wedge b=0$. Then we have $a^{\perp} \wedge b=b$. It follows that $b=0$.

Now, assume that if $a \wedge b=0$, then $b=0$. Since $a \wedge a^{*}=0, a^{*}=0$.

5) Since $a^{\perp}, a^{*} \wedge 0^{\perp} \leq 0^{\perp}$, we get $a^{\perp} \wedge\left(a^{*} \wedge 0^{\perp}\right)=\left(a^{*} \wedge 0^{\perp}\right) \wedge a^{\perp}$. Now, since $a \wedge\left(a^{*} \wedge a^{\perp}\right)=0, a^{\perp} \wedge\left(a^{*} \wedge 0^{\perp}\right)=a^{*} \wedge 0^{\perp}$.

Again, since $0 \wedge a^{\perp}=0,0^{\perp} \wedge a^{\perp}=a^{\perp}$. Therefore $\left(a^{*} \wedge 0^{\perp}\right) \wedge a^{\perp}=a^{*} \wedge\left(0^{\perp} \wedge\right.$ $\left.a^{\perp}\right)=a^{*} \wedge a^{\perp}=a^{\perp}$. It follows that, $a^{*} \wedge 0^{\perp}=a^{\perp}$.

Now, we prove the following.

Theorem 4.4. Let $L$ be an AL with 0 and $*$ be a pseudo-complementation on $L$. Let $M$ be the set of all maximal elements in $L$ and let $P C(L)$ be the set of all pseudo complementation on $L$. For any $m \in M$, define $*_{m}: L \rightarrow L$ by $a^{*} m=a^{*} \wedge m$ for all $a \in L$. Then $m \mapsto *_{m}$ is a bijection of $M$ onto $P C(L)$.

Proof: Let $m, n \in M$ such that $*_{m}=*_{n}$. Then $0^{*} m=0^{*} n$. This implies $0^{*} \wedge m=0^{*} \wedge$ $n$. Hence, we get $m=n$. Also, for any $\perp \in P C(L)$, if $m=0^{\perp}$, then $a^{*} m=a^{*} \wedge m=$ $a^{*} \wedge 0^{\perp}=a^{\perp}$. Then $\perp$ is the same as $*_{m}$ and $m$ is maximal. Thus $m \mapsto *_{m}$ is a bijection of $M$ onto $P C(L)$.

Finally, we prove that the Boolean algebra $L^{*}$ is independent (upto isomorphism) of the pseudo-complementation $*$ on $L$ in the following.

Theorem 4.5. Let $L$ be an AL with 0 and let $*, \perp$ be two pseudo-complementations on $L$. Then the Boolean algebras $L^{*}$ and $L^{\perp}$ are isomorphic.

Proof: Proof follows by lemma 4.3., by the definition of pseudo-complementation and by lemma 3.11.

\section{REFERENCES}

1. A.F.Lopez and M.I.T.Barroso, Pseudo-complemented semilattices, boolean algebras, and compatible products, Journal of Algebra, 242 (2001) 60-91.

2. M.Ayub Ali, R.M.H.Rahman and A.S.A.Noor, Some properties of semi-prime ideals in lattices, Annals of Pure and Applied Mathematics, 1(2) (2012) 176-185.

3. G.Birkhoff, Lattice theory, 3rd ed., Amer. Math. Soc. Colloq. Publ., vol. 25, Amer. Math. Soc., Providence, R. I., 1967. MR 37 \#2638.

4. T.S.Blyth, Ideals and filters of pseudo-complemented semilattices, Proceeding of the Edinburgh Mathematical Society, 23 (1980) 301-316.

5. W.H.Cornish, Congruences on distributive pseudo-complemented lattices, Bull. Austral. Math. Soc., 8 (1973) 161-179.

6. O.Frink, Pseudo-complementations in semi-lattice, Duke Math. J., 29 (1962) 505514. 
Pseuduo-Complementation on Almost Lattices

7. T. Katriňák and J. Guričan, Finite pseudocomplemented lattices: The spectra and the Glivenko congruence, Algebra Universalis, 66(1-2) (2011) 151-161.

8. H.Lakser, The structure of Pseudocomplemented distributive lattices I: Subdirect decomposition, Transaction of the American Mathematical Society, 156 (1971) 335342.

9. K.B.Lee, Equational class of distributive pseudo-complemented lattice, Cand. J. Math., 22 (1970) 881-891.

10. G.Nanaji Rao and H.T.Alemu, Almost lattices, J. Int. Mat. Virt. Inst., 9 (2019) 151171.

11. G.Nanaji Rao and Sujatha Kumari, Pseudo-complemented almost semilattices, International Journal of Mathematical Archive, 8(10) (2017) 94-102.

12. P.Ribenboim, Characterization of the pseudo-complement in a distributive lattice with least element, Summa Brasil. Math., 2(1949) 43-49.

13. T.P.Speed, Some remarks on a class of distributive lattice, Jour. Aust. Math. Soc., 9 (1969) 289-296.

14. T.P.Speed, On stone lattices, J. Aust. Math. Soc., 9 (1969) 297-307.

15. U.M.Swamy and P.Manikyamba, Prime ideal characterization of stone lattices, Math Seminar Notes, 7 (1979) 25-31.

16. G.Szasz, Introduction to lattice theory, Academic Press, New York and London (1963).

17. J.C.Varlet, A generalization of the notion of pseudo-complementedness, Bull. Soc. Sci. Liege, 37 (1968) 149-158.

18. P.V.Venkatanarasimhan, Pseudo-complements in posets, Proceedings of the American Mathematical Society, 28 (1971) 9-17. 